\title{
INVARIANT THEORY OF $G_{2}$
}

\author{
BY GERALD W. SCHWARZ ${ }^{1}$
}

Introduction. Let $V$ denote $\mathbf{C}^{n}$, and let $G \subseteq \mathrm{SL}(V)$ be a classical subgroup. Then Classical Invariant Theory (CIT) describes the generators and relations of the algebra of invariant polynomial functions $\mathbf{C}[m V]^{G}$, where $m \in \mathbf{Z}^{+}$and $m V$ denotes the direct sum of $m$ copies of $V$. Using the symbolic method (see [7]), one can then obtain a handle on the invariants of arbitrary representations of $G$. These classical methods and results have been very useful in many areas of mathematics.

Let $G$ be a connected, simple, and simply connected complex algebraic group. Then $G$ is classical except when $G=\operatorname{Spin}_{n}, n \geq 7$, or in case $G$ is an exceptional group $G_{2}, F_{4}, E_{6}, E_{7}$, or $E_{8}$. It would be useful to have an analogue of CIT for nonclassical $G$. We have succeeded in establishing an analogue for $G_{2}$ (described below). We also have a conjectured analogue for $\mathrm{Spin}_{7}$, but a complete proof requires a computation we are as yet unable to perform.

The Cayley algebra, $G_{2}$, and the Main Theorem. Let Cay denote the usual (complex) Cayley algebra (see [3]). Then Cay is a nonassociative, noncommutative algebra of dimension 8 over $\mathbf{C}$. Let $\mathrm{Cay}^{\prime}$ denote the (7dimensional) span of all commutators of elements of Cay. Let $\operatorname{tr}:$ Cay $\rightarrow \mathbf{C}$ denote the linear map with kernel Cay' which sends $1 \in$ Cay to $1 \in \mathbf{C}$. Define $\bar{x}=-x+2 \operatorname{tr}(x) \cdot 1, x \in$ Cay. Then $x \mapsto \bar{x}$ is an involution such that $x \bar{x}=$ $n(x) \cdot 1 \in \mathbf{C} \cdot 1$ for all $x \in$ Cay. Moreover,

$$
\begin{gathered}
x(x y)=x^{2} y ; \quad(y x) x=y x^{2}, \quad x, y \in \text { Cay. } \\
x^{2}-2 \operatorname{tr}(x) x+n(x) \cdot 1=0, \quad x \in \text { Cay. } \\
x \mapsto n(x) \text { is a nondegenerate quadratic form on Cay. }
\end{gathered}
$$

The identities in (1), called the alternative laws, are a weak form of associativity. Equation (2) is called the standard quadratic identity.

$G_{2}$ is the group of algebra automorphisms of Cay. Thus $G_{2}$ acts trivially on $\mathbf{C} \cdot 1$ and faithfully (and orthogonally) on Cay'. From now on, let $G$ denote $G_{2}$ and let $V$ denote Cay'. By (3), $V$ is $G$-isomorphic to its dual $V^{*}$.

The following is our main result.

Received by the editors April 25, 1983.

1980 Mathematics Subject Classification. Primary 17A36, 20F29, 20 G05.

${ }^{1}$ Research partially supported by NSF grant MCS 7902937. 
ThEOREM 4. Let $m \in \mathbf{Z}^{+}$, and let $x_{j}$ denote a typical element in the $j$ th copy of $V$ in $m V, 1 \leq j \leq m$.

(4.1) $\mathbf{C}[m V]^{G}$ is generated by elements $\operatorname{tr}\left(x_{i_{1}}\left(x_{i_{2}} \cdots x_{i_{r}}\right) \cdots\right), r \leq 4$.

(4.2) The relations of these generators are consequences of identities (1) and (2). Moreover, the relations are generated by ones of degrees 6,7, and 8 in the $x_{j}$.

In (4.1) the elements of $V=$ Cay' $^{\prime}$ are multiplied in Cay; the traces of such products are clearly $G$-invariant. The relations of (4.2) are obtained by replacing $x$ and $y$ in (1) and (2) by products of elements of Cay', multiplying the resulting equations by other products, and then taking traces.

Our results are analogous to those of Kostant-Procesi-Rasmyslev (see [2]) for the adjoint representation of $\mathrm{SL}_{n}$. In their case (1) is replaced by associativity, and (2) by the Cayley-Hamilton identity.

As outlined below, we determined generators and relations for $\mathbf{C}[m V]^{G}$ using techniques of invariant theory and commutative algebra. We then showed, a posteriori, that the generators and relations are as in (4.1) and (4.2). J. Ferrar has informed us that he also has a proof of (4.1). Set

Generators. We sketch a proof of (4.1): Let $x_{1}, \ldots, x_{m}$ be as in the Theorem.

where in (5.3) we skew symmetrize in the indices. The invariant $\alpha_{i j}$ is symmetric in its indices, while $\beta_{i j k}$ and $\gamma_{i j k l}$ are skew symmetric in theirs (hence are zero if the same index appears twice).

Let $\omega$ denote a nonzero element of $\left(\bigwedge^{3} V\right)^{G}$ corresponding to the $\beta$ type invariants. One can show that wedge multiplication by $\omega$ gives an isomorphism of $\bigwedge^{2} V$ with $\bigwedge^{5} V$, and it follows that generators of $\mathbf{C}[m V]^{G}$ can be obtained by polarization from those in the case $m=4$. A theorem of Weyl [7, p. 154] says that, when $m=4$, it suffices to consider generators whose degree $d$ in the fourth copy of $V$ is at most 1 . These generators correspond to invariants in $\mathbf{C}[3 V]$ (if $d=0$ ), and copies of $V^{*}=V$ in $\mathbf{C}[3 V]$ (if $d=1$ ). Using [4] and [5] one can show that $\mathbf{C}[3 V]^{G}$ is generated by $\alpha$ and $\beta$ type invariants, and that the covariants in $\mathbf{C}[3 V]$ corresponding to the representation $V$ form a free $\mathbf{C}[3 V]^{G}$-module with three generators in degree 1 , three in degree 2 , and one in degree 3 . The degree 3 generator corresponds to an invariant of type $\gamma$; the other generators give nothing new. Thus $\mathbf{C}[m V]^{G}$ is generated by invariants of types $\alpha, \beta$, and $\gamma$; establishing (4.1).

Relations (Proof of (4.2)). Since $V \simeq V^{*}$, we can just as well consider computing the $G$-invariants of the symmetric algebra $S^{*}(m V)$. Note that $\mathrm{GL}_{m}$ acts naturally on $S^{*}(m V)^{G} \simeq S^{*}\left(V \otimes \mathbf{C}^{m}\right)^{G}$.

Let $\phi_{i}$ denote the standard representation of $\mathrm{GL}_{m}$ on $\wedge^{i} \mathbf{C}^{m}$ (so $\phi_{i}=0$ if $i>m$ ). If $a_{1}, \ldots, a_{k} \in \mathbf{Z}^{+}$, let $\phi=\phi_{1}^{a_{1}} \cdots \phi_{k}^{a_{k}}$ denote the highest weight component of $S^{a_{1}}\left(\phi_{1}\right) \otimes \cdots \otimes S^{a_{k}}\left(\phi_{k}\right)$. If $a_{k}>0$, we say that $\phi$ has height $k$. 
The generators of $S^{*}\left(V \otimes \mathbf{C}^{m}\right)^{G}$ of (5.1), (5.2), and (5.3) transform by the representations $\phi_{1}^{2}, \phi_{3}$, and $\phi_{4}$, respectively. Thus there is a $\mathrm{GL}_{m}$-equivariant surjection $\pi$ from $R=S^{*}\left(\phi_{1}^{2}+\phi_{3}+\phi_{4}\right)$ to $S=S^{*}\left(V \otimes \mathbf{C}^{m}\right)^{G}$, and $I=\operatorname{Ker} \pi$ is $\mathrm{GL}_{m}$-invariant and homogeneous (grade $R$ and $S$ in the obvious way). Comparing $R$ and $S$ in degrees $\leq 8$, one finds the following elements of $I$ (see explanation below).

$$
\begin{array}{ll}
\phi_{1} \phi_{5} \subseteq S^{2} \phi_{3} ; & \phi_{1} \phi_{5} \subseteq \phi_{1}^{2} \otimes \phi_{4}, \\
\phi_{2} \phi_{5} \subseteq \phi_{3} \otimes \phi_{4} ; & \phi_{2} \phi_{5} \subseteq S^{2} \phi_{1}^{2} \otimes \phi_{3}, \\
\phi_{1} \phi_{6} \subseteq \phi_{3} \otimes \phi_{4}, & \\
\phi_{8} \subseteq S^{2} \phi_{4}, & \\
\phi_{2} \phi_{6} \subseteq S^{2} \phi_{4} ; & \phi_{2} \phi_{6} \subseteq S^{2} \phi_{1}^{2} \otimes \phi_{4}, \\
\phi_{4}^{2} \subseteq S^{2} \phi_{4} ; & \phi_{4}^{2} \subseteq S^{4} \phi_{1}^{2} ; \quad \phi_{4}^{2} \subseteq \phi_{1}^{2} \otimes S^{2} \phi_{3} .
\end{array}
$$

Each relation consists of a nontrivial "linear combination" of the given representations in $R$ whose image is zero in $S$. For example, in (6.1), a highest weight vector of the space of relations is $\sigma+\tau$, where

$$
\sigma=\beta_{123} \beta_{145}-\beta_{124} \beta_{135}+\beta_{125} \beta_{134}
$$

is a highest weight vector of $\phi_{1} \phi_{5} \subseteq S^{2} \phi_{3}$, and

$$
\tau=\alpha_{11} \gamma_{2345}-\alpha_{12} \gamma_{1345}+\alpha_{13} \gamma_{1245}-\alpha_{14} \gamma_{1235}+\alpha_{15} \gamma_{1234}
$$

is a highest weight vector of $\phi_{1} \phi_{5} \subseteq \phi_{1}^{2} \otimes \phi_{4}$.

It is not difficult to show that relations (6.1)-(6.6) generate $I$ for any $m$ if they generate in the case $m=6$ (this has a lot to do with the fact that $\operatorname{dim} V=7$ !), so we may assume $m=6$. Using techniques of [5] one can show that $S$ has a regular sequence $f_{1}, \ldots, f_{28}$ consisting of 18 forms of degree 2 and 10 forms of degree 3. Since $S$ is Cohen-Macaulay (even Gorenstein [1, p. 124]) of dimension 28 , we find that $S \simeq \mathbf{C}\left[f_{1}, \ldots, f_{28}\right] \otimes S^{0}$ (as graded C $\left[f_{1}, \ldots, f_{28}\right]$-module), where $S^{0}=S /\left(f_{1}, \ldots, f_{28}\right)$ is an artin algebra. Thus the Poincaré series $P(t)$ of $S$ equals $\left(1-t^{2}\right)^{-18}\left(1-t^{3}\right)^{-10} P^{0}(t)$, where $P^{0}(t)=$ $\sum_{i=0}^{l} a_{i} t^{i}$ is the Poincaré series for $S^{0}$. Since $S$ is Gorenstein, $a_{i}=a_{l-i}, 0 \leq$ $i \leq l$, and using a result of Stanley [6] one can show that $l=24$. Since $S$ has generators of degree $\leq 4$, it follows that $I$ is generated by elements of degree $\leq l+4=28$. Thus we have to show that (6.1)-(6.6) generate $I$ in degrees $\leq 28$. This computation was not easy to do, but was made manageable by the $\mathrm{GL}_{6}$ symmetry and certain estimates arising out of (6.1)-(6.6).

Details are to appear.

\section{REFERENCES}

1. M. Hochster and J. Roberts, Rings of invariants of reductive groups acting on regular rings are Cohen-Macaulay, Adv. in Math. 13 (1974), 115-175.

2. C. Procesi, The invariant theory of $n \times n$ matrices, Adv. in Math. 19 (1976), 306-381.

3. R. D. Schafer, An introduction to non-associative algebras, Academic Press, New York, 1966.

4. G. Schwarz, Representations of simple Lie groups with regular rings of invariants, Invent. Math. 49 (1978), 167-191. 
5. __ Representations of simple Lie groups with a free module of covariants, Invent. Math. 50 (1978), 1-12.

6. R. P. Stanley, Combinatorics and invariant theory, Proc. Sympos. Pure Math., Vol. 34, Amer. Math. Soc., Providence, R. I., 1979, pp. 345-355.

7. H. Weyl, The classical groups, 2nd ed., Princeton Univ. Press, Princeton, N. J., 1946.

Department of Mathematics, Brandeis University, Waltham, MassachuSETTS 02254 